\title{
DESIGN OF SECURE AND RELIABLE MU-MiMo TRANSCEIVER SYSTEM FOR VEHICULAR NETWORKS
}

\author{
C. Manikandan, P. Neelamegam, S.Rakesh Kumar, G. Venkat Babu, and Sai Siva \\ Satwik Kommi
}

\begin{abstract}
School of Electrical and Electronics Engineering, SASTRA Deemed University, Thanjavur, Tamil Nadu, India.
\end{abstract}

\begin{abstract}
MU-MIMO (Multi-User MIMO) has been a promising technique for vehicular networks to achieve faster communication. Conventional MU-MIMO transceiver is designed with One-dimensional (1-D) improper modulation schemes such as Binary Phase Shift Keying (BPSK) and Multilevel Amplitude Shift Keying (M$A S K)$ failed to yield standard ABER (average bit error rate). To achieve high reliability, a novel MUMIMO uplink transceiver system is designed under PAPC (Per-Antenna Power Constraint) by assuming perfect and imperfect channel state information (CSI). MIMO communication channels are perceptible. Hence, security of the proposed system is improved by novel pseudorandom key generation technique using randomized synthetic colour image. Analytical design for proposed systems is carried and simulated for various p-norm constraints. Simulation results show higher reliability and security than the existing system. It also satisfies the linearity constraint of a power amplifier, which makes the system more suitable for practical applications.
\end{abstract}

\section{KEYWORDS}

MU-MIMO, Uplink, PAPC, 1-D improper modulation, Perfect \& imperfect CSI, Colour Image, Pseudorandom key generation

\section{INTRODUCTION}

Vehicular networks are widely used for autonomous navigation, remotely operated vehicle, and swarm robot [1]. It utilizes cellular and wireless local area networks and demands high-speed, reliable communication without increasing channel bandwidth and power required for transmission [2-4]. To meet such requirement, MU-MIMO communication technology has been introduced in such applications [5]. Several MU-MIMO transceivers design has been reported to increase throughput and minimize total mean square error (TMSE). In all the cases a common transceiver design has been proposed for both proper and improper modulation [6-13]. The outcome revealed that the Average Bit error rate (ABER) performance of existing MU-MIMO transceiver systems was suboptimal for improper modulation. To overcome this problem, a separate MU-MIMO transceiver system had been designed for improper modulations with novel precoding strategy [14]. Joint optimal precoders and decoders were designed for improper modulation to improve the ABER performance [15-18].

Predominately the exiting MU-MIMO transceiver systems were designed under TPC ( $\beta$ ). In TPC based power allocation techniques, linearity constraint of the individual power amplifier is not considered. This makes the system fail to meet the practical requirements and makes its challenging to be realized in real-time. An SU-MIMO transceiver has been proposed with 1-D improper modulation and PAPC [19] to meet the practical requirements in point-to-point communication. This has been extended for MU-MIMO uplink transceiver system. Additionally, to provide security, a simple and efficient novel key generation algorithm has been developed 
using randomized synthetic colour image. This makes the proposed system to have high data rate and reliability with security, which are the primary requirements for vehicular network.

The remaining sections of this article are organized as follows: Section 2 illustrates the system model used for uplink vehicular network and describes the design procedure of the proposed MUMIMO transceiver systems and its key generation techniques. In section 3 numerical results of the proposed system are provided. Section 4 concludes the paper with future directions.

\section{SYSTEM MODEL}

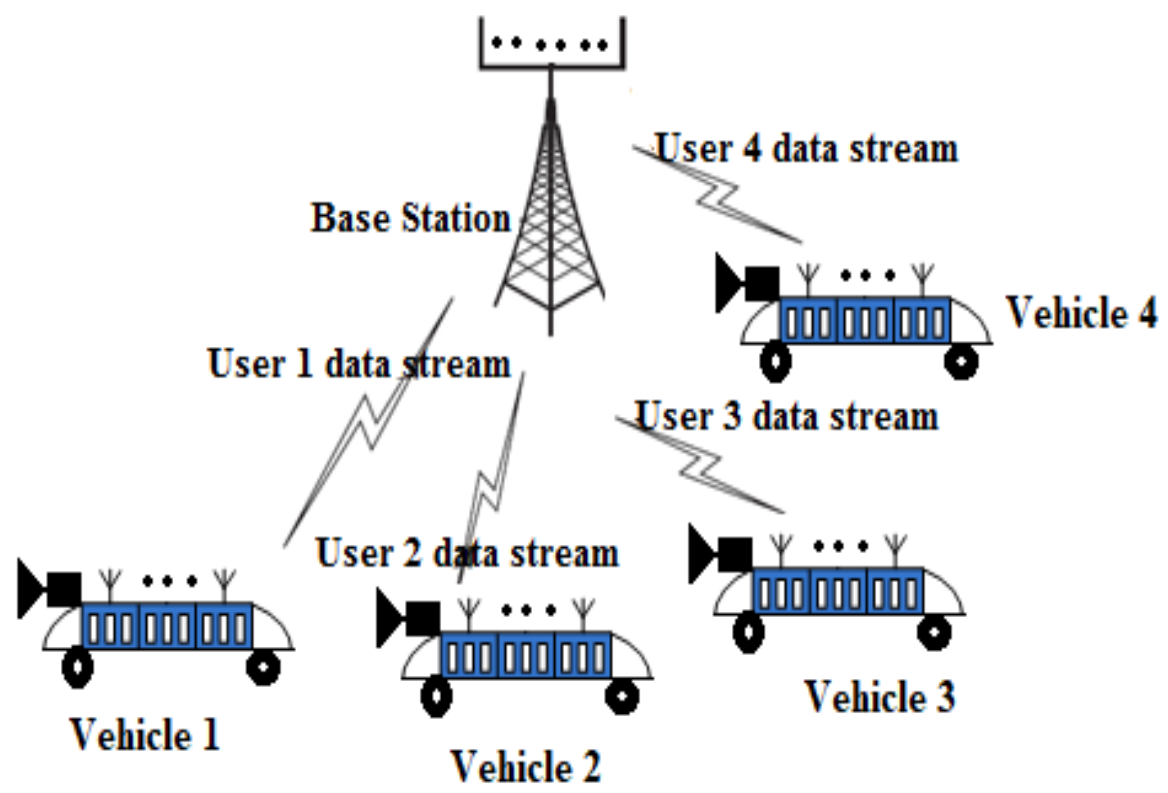

Figure 1. MU-MIMO Vehicular system models

The vehicular network provides reliable communication between the individual vehicles and the base station [1]. In the proposed work, uplink MU-MIMO transceiver is implemented in infrastructure-based vehicular networks. It consists of a single Base Station (BS) and four Vehicular Nodes (VNs) as shown in Figure 1. Both the BS and the VNs are equipped with multiple antennas. Each VNs connects to the base station with an independent uplink channel $\mathrm{H}_{\mathrm{m}}{ }^{\text {(UL) }}$ where $\mathrm{m}=1,2 \ldots 4$. All the vehicles utilize the same frequency spectrum for signal transmission. However, they are separated in the spatial domain. Vision sensor attached at frontend of each VNs simultaneously transmits its information to BS through $\mathrm{H}_{\mathrm{m}}{ }^{(\mathrm{UL})}$. After receiving the information, BS generates a command to navigate multiple vehicles on the road. 


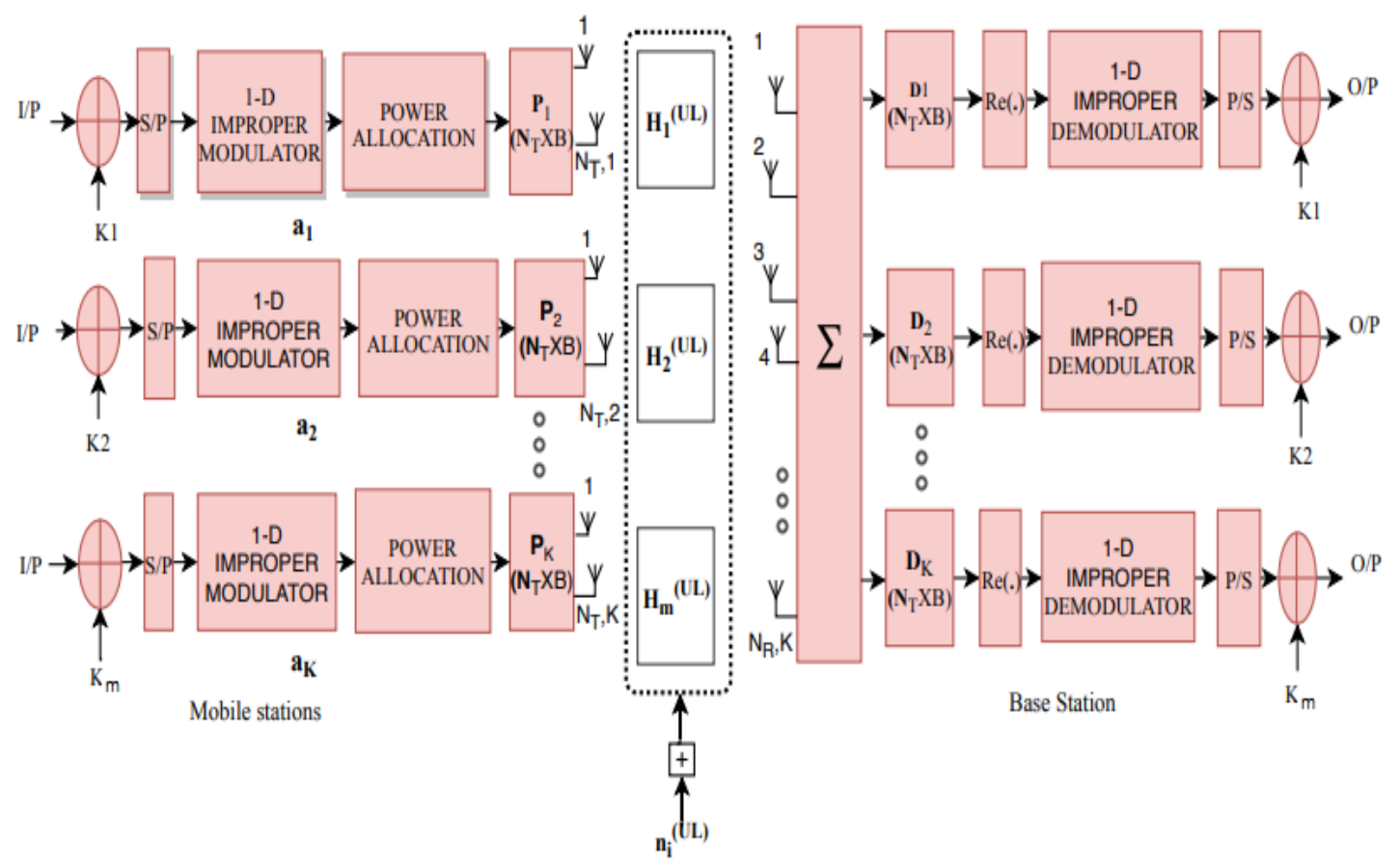

Figure 2. Proposed MU-MIMO uplink system

The block diagram of the proposed MU-MIMO uplink transceiver system with ' $\mathrm{m}$ ' channels is shown in Figure 2. For the encryption process, the analog data obtained from the sensor is converted into binary and XORed with a key to get cipher text. This encrypted plain text is converted to parallel form and modulated using 1-D improper modulator for transmission. Digital modulation scheme yielding improper signal is called as improper modulation. In the proposed linear MIMO transceiver system improper modulation such as BPSK and M-ary ASK was implemented its pseudo-autocorrelation and cross-correlation function of complex envelope signals are assumed as nonzero [18]. A linear precoder concerning PAPC $(\alpha)$ is used to encode data stream of each VNs. Since the uplink channel matrices are complex, the coded data streams are multiplied by complex channel matrix $\left(\mathrm{H}_{\mathrm{m}}{ }^{(\mathrm{UL})}\right)$ and decoded by the linear decoders at the receiver. As the modulator outputs consist of only real components. Hence the real part of decoder output is considered for detection. The precoder and decoder matrix is updated based on the assumption of perfect and imperfect channel state information known at the BS and VN. 


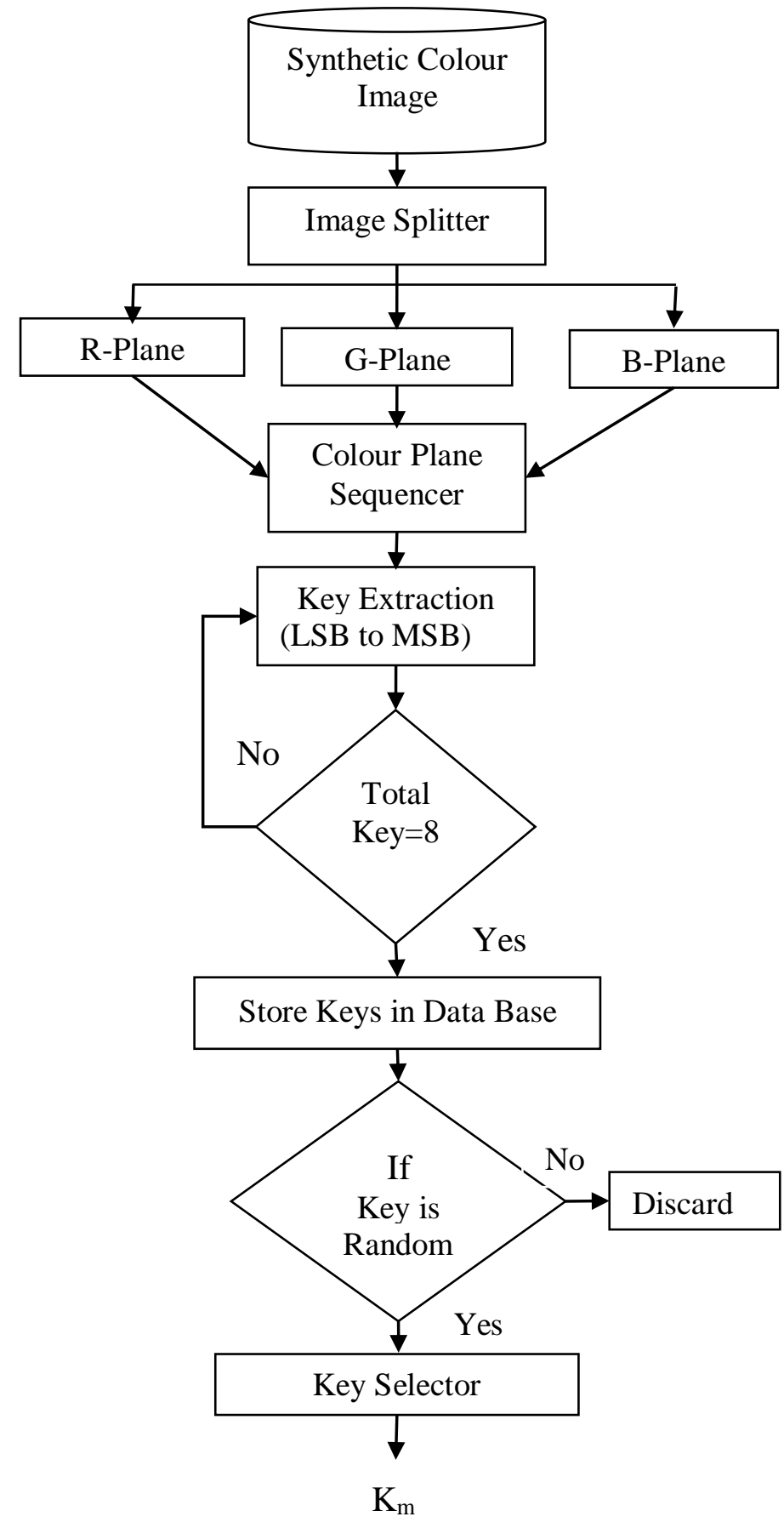

Figure.3. Flow Chart for key generation algorithm

A novel image-based key generation technique proposed for the stream cipher encryption and decryption algorithm is described in Figure.3. A 256×256 random pixel synthetic colour image is chosen as shown in Figure. 4. The colour image is made up of three layers namely red, green and blue. The pixel values in each layer vary from 0 to 255 representing the intensity level. Therefore, 8 bits are required to represent the value of one pixel. This facilitates the generation of 8 separate keys with a size of 65536 bits by extracting one bit at a time from LSB to MSB as shown in Figure.5. 
The key generation process is repeated for all the three layers, and a total of 24 keys are generated. The keys fulfill the randomness property are used for encryption process, and others are discarded. To get better protection against brute force attacker, a key selection process for encryption and decryption also performed randomly.

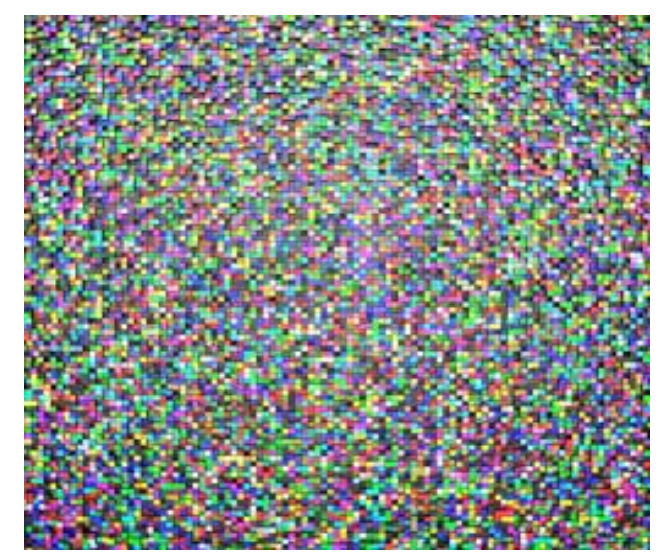

Figure. 4 Random pixel synthetic colour image

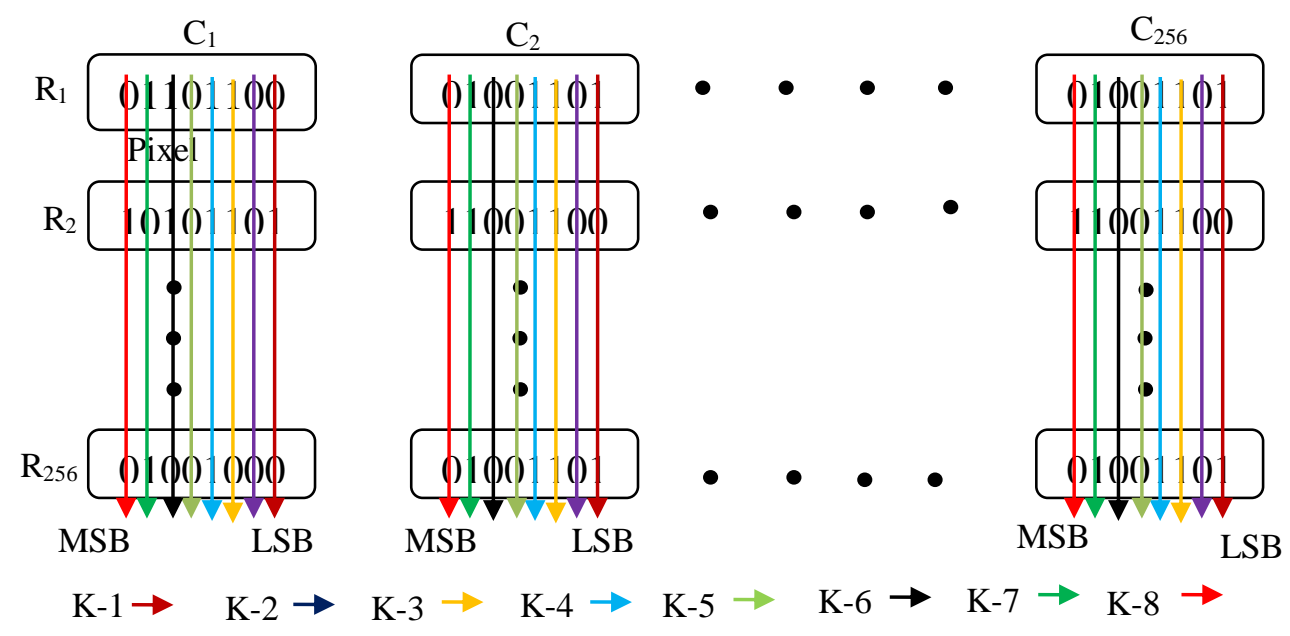

Figure. 5 Method for Key Generation

\subsection{Problem formulation for minimum TMSE design with perfect CSI}

The mathematical model for uplink MU-MIMO system by assuming channel matrix $\mathrm{H}$ is perfectly known to both transmitter and receiver is derived as follows. This model is used to minimize the TMSE for the detected symbol. The notations and their meanings used in this paper are given in Table 1. 
International Journal of Computer Networks \& Communications (IJCNC) Vol.11, No.2, March 2019

Table 1. Notations and their meanings used in the system model

\begin{tabular}{|l|l|}
\hline Notation & Meaning \\
\hline $\mathrm{K}_{\mathrm{m}}$ & $\mathrm{K}_{\mathrm{m}}$ is a randomly chosen key from the finite set of possible keys \\
\hline $\mathbf{d}_{l}^{\wedge}(U L)$ & Output data vectors form the $l^{\text {th }}$ decoder \\
\hline $\mathbf{a}_{l}$ & Input data vectors to the $l^{\text {th }}$ precoder \\
\hline $\mathbf{H}_{m}^{\wedge}(U L)$ & Uplink MIMO channel matrices of $m^{\text {th }}$ vehicle \\
\hline $\mathbf{n}_{l}^{(U L)}$ & $\begin{array}{l}\text { Additive White Gaussian Noise (AWGN) vector with zero-mean and } \\
\text { variance }\left(\sigma_{n}^{(U L)}\right)^{2} \cdot \text { whose entries are spatially, temporally and } \\
\text { independent and identically distributed (i.i.d) }\end{array}$ \\
\hline $\mathbf{P}_{m}$ & Precoding matrix for the $m^{\text {th }}$ Vehicle \\
\hline $\mathbf{D}_{l}$ & Decoding matrix for the $l^{\text {th }}$ Vehicle \\
\hline $\mathrm{B}$ & TPC \\
\hline $\mathrm{A}$ & PAPC \\
\hline$\|\cdot\|$ & p-norm norm \\
\hline $\mathrm{E}(\cdot)$ & Expectation \\
\hline $\mathrm{R}(\cdot)$ & Real part of a complex-valued vector \\
\hline$(\cdot)^{-1}$ & Matrix inverse \\
\hline$(\cdot)^{\mathrm{T}}$ & Matrix transpose \\
\hline$(\cdot)^{*}$ & Matrix complex conjugate \\
\hline$(\cdot)^{\mathrm{H}}$ & Matrix Hermitian \\
\hline $\operatorname{Tr}(\cdot)$ & Trace of a matrix \\
\hline $\mathbf{I}_{\mathrm{B}}$ & Identity matrix, \\
\hline $\mathrm{N}_{\mathrm{T}}$ & Number of transmit antennas \\
\hline $\mathrm{N}_{\mathrm{R}}$ & Number of receive antennas \\
\hline $\mathrm{B}_{\mathrm{m}}$ & Data stream for the $m^{\text {th }}$ Vehicle \\
\hline
\end{tabular}

The TMSE matrix for uplink can be formulated as,

$E\left[\left\|\mathbf{e}^{(U L)}\right\|^{2}\right]=E\left[\left\|\mathbf{d}_{l}^{\wedge(U L)}-\mathbf{a}_{l}\right\|^{2}\right]$

where $\quad \mathbf{d}_{l}^{\wedge(U L)}=R\left(\mathbf{D}_{l}\left[\sum_{m=1}^{K} \mathbf{H}_{m}^{(U L)} \mathbf{P}_{m} \mathbf{a}_{m}\right]+\mathbf{D}_{l} \mathbf{n}_{l}^{(U L)}\right)$

Substituting the value of $\mathbf{d}_{l}^{\wedge(U L)}$ in (1), we obtain

$$
E\left[\left\|\mathbf{e}^{(U L)}\right\|^{2}\right]=E\left[\left\|R\left(\mathbf{D}_{l}\left[\sum_{m=1}^{K} \mathbf{H}_{m}^{(U L)} \mathbf{P}_{m} \mathbf{a}_{m}\right]+\mathbf{D}_{l} \mathbf{n}_{l}^{(U L)}\right)-\mathbf{a}_{l}\right\|^{2}\right]
$$

The TMSE calculation is expanded as follows: 
International Journal of Computer Networks \& Communications (IJCNC) Vol.11, No.2, March 2019

$$
\begin{aligned}
& E\left[\left\|\mathbf{e}^{(U L)}\right\|^{2}\right]=\operatorname{Tr}\left\{E \left(0.5\left(\mathbf{D}_{l} \sum_{m=1}^{K} \mathbf{H}_{m}^{(U L)} \mathbf{P}_{m} \mathbf{a}_{m}+\mathbf{D}_{l}^{*} \sum_{m=1}^{K}\left(\mathbf{H}_{m}^{(U L)}\right)^{*} \mathbf{P}_{m}^{*} \mathbf{a}_{m}^{*}\right)+0.5\left(\mathbf{D}_{l} \mathbf{n}_{l}^{(U L)}+\mathbf{D}_{l}^{*}\left(\mathbf{n}_{l}^{(U L)}\right)^{*}\right)\right.\right. \\
& \left.-\sum_{m=1}^{K} \mathbf{a}_{m}\right)\left(0 . 5 \left(\mathbf{D}_{l}^{\mathrm{H}} \sum_{m=1}^{K}\left(\mathbf{H}_{m}^{(U L)}\right)^{\mathrm{H}} \mathbf{P}_{m}^{\mathrm{H}} \mathbf{a}_{m}^{\mathrm{H}}+\mathbf{D}_{l}^{\top} \sum_{m=1}^{K}\left(\mathbf{H}_{m}^{(U L)}\right)^{\top} \mathbf{P}_{m}^{\top} \mathbf{a}_{m}^{\top}+0.5\left(\mathbf{D}_{l}^{\mathrm{H}}\left(\mathbf{n}_{l}^{(U L)}\right)^{\mathrm{H}}+\mathbf{D}_{l}^{\top}\left(\mathbf{n}_{l}^{(U L)}\right)^{\top}\right)\right.\right. \\
& \left.\left.-\sum_{m=1}^{K} \mathbf{a}_{m}^{\mathrm{H}}\right)\right\}
\end{aligned}
$$

Using assumptions made in [15-18], equation (3) is simplified for calculation of TMSE

$$
\begin{aligned}
& E\left[\left\|\mathbf{e}^{(U L)}\right\|^{2}\right]=\operatorname{Tr}\left\{0.25 \mathbf{D}_{l} \sum_{m=1}^{K} \mathbf{H}_{m}^{(U L)}\left(\mathbf{H}_{m}^{(U L)}\right)^{\top} \mathbf{P}_{m} \mathbf{P}_{m}^{\top} \mathbf{D}_{l}^{\top}+0.25 \mathbf{D}_{l} \sum_{m=1}^{K} \mathbf{H}_{m}^{(U L)}\left(\mathbf{H}_{m}^{(U L)}\right)^{\mathrm{H}} \mathbf{P}_{m} \mathbf{P}_{m}^{\mathrm{H}} \mathbf{D}_{l}^{\mathrm{H}}\right. \\
& +0.25 \mathbf{D}_{l}^{*} \sum_{m=1}^{K}\left(\mathbf{H}_{m}^{(U L)}\right)^{*}\left(\mathbf{H}_{m}^{(U L)}\right)^{\top} \mathbf{P}_{m}^{*} \mathbf{P}_{m}^{\top} \mathbf{D}_{l}^{\top}+0.25 \mathbf{D}_{l}^{*} \sum_{m=1}^{K}\left(\mathbf{H}_{m}^{(U L)}\right)^{*}\left(\mathbf{H}_{m}^{(U L)}\right)^{\mathrm{H}} \mathbf{P}_{m}^{*} \mathbf{P}_{m}^{\mathrm{H}} \mathbf{D}_{l}^{\mathrm{H}} \\
& -0.5 \mathbf{D}_{l} \sum_{m=1}^{K} \mathbf{H}_{m}^{(U L)} \mathbf{P}_{m}-0.5 \mathbf{D}_{l}^{*} \sum_{m=1}^{K}\left(\mathbf{H}_{m}^{(U L)}\right)^{*} \mathbf{P}_{m}^{*}-0.5 \mathbf{D}_{l}^{\top} \sum_{m=1}^{K}\left(\mathbf{H}_{m}^{(U L)}\right)^{\top} \mathbf{P}_{m}^{\top}-0.5 \mathbf{D}_{l}^{\mathrm{H}} \sum_{m=1}^{K}\left(\mathbf{H}_{m}^{(U L)}\right)^{\mathrm{H}} \mathbf{P}_{m}^{\mathrm{H}} \\
& \left.+0.25\left(\sigma_{n, l}^{(U L)}\right)^{2} \mathbf{D}_{l} \mathbf{D}_{l}^{\mathrm{H}}+0.25\left(\sigma_{n, l}^{(U L)}\right)^{2} \mathbf{D}_{l}^{*} \mathbf{D}_{l}^{\top}+\mathbf{I}_{B_{l}}\right\}
\end{aligned}
$$

To minimize the TMSE subject to PAPC,

$$
\min _{\mathbf{P}_{\mathbf{m}}, \mathbf{D}_{\mathbf{m}}} \operatorname{tr}\left(E\left[\left\|\mathbf{e}^{(U L)}\right\|^{2}\right]\right) \text { s.t. } \sum_{m=1}^{K}\left(\operatorname{Tr}\left(\mathbf{P}_{m} \mathbf{P}_{m}^{\mathrm{H}}\right)^{p}\right)^{1 / p} \leq \alpha
$$

Where $\alpha$ is the PAPC.

Using Lagrangian to get the solution for the defined problem

$$
\eta^{(U L)}=E\left[\left\|\mathbf{e}^{(U L)}\right\|^{2}\right]+\mu^{(U L)}\left(\left[\sum_{m=1}^{K}\left(\operatorname{Tr}\left(\mathbf{P}_{m} \mathbf{P}_{m}^{\mathrm{H}}\right)^{p}\right)^{1 / p}\right]-\alpha\right)
$$

where $\mu^{(U L)}$ is the Lagrange multiplier.

Substitute equation (4) in (6) to get an expanded version of (6). The uplink one-dimensional improper modulation based transceiver design problem in (5) is non-convex and also continuously differentiable. Therefore by taking the derivatives of $\eta^{(U L)}$ with respect to $\mathbf{D}_{l}$ and $\mathbf{P}_{l}$, Then the related Karush-Kuhn-Tucker(KKT) conditions can be obtained and given in the following

$$
\begin{aligned}
& \frac{\partial \eta^{(U L)}}{\partial \mathbf{D}_{l}}=0 \\
& \mathbf{D}_{z}\left\{\sum_{z=1}^{K} \mathbf{H}_{Z}^{(U L)} \mathbf{P}_{z} \mathbf{P}_{z}^{\mathrm{H}} \mathbf{H}_{z}^{(U L)^{\mathrm{H}}}+\left(\sigma_{n}^{(U L)}\right)^{2}\right\}+\mathbf{D}_{z}^{*}\left\{\sum_{z=1}^{K}\left(\mathbf{H}_{Z}^{(U L)}\right)^{*} \mathbf{P}_{z}^{*} \mathbf{P}_{z}^{\mathrm{H}}\left(\mathbf{H}_{Z}^{(U L)}\right)^{\mathrm{H}}\right\}=2 \mathbf{P}_{z}^{\mathrm{H}}\left(\mathbf{H}_{Z}^{(U L)}\right)^{\mathrm{H}} \\
& \frac{\partial \eta^{(U L)}}{\partial \mathbf{P}_{l}}=0
\end{aligned}
$$


International Journal of Computer Networks \& Communications (IJCNC) Vol.11, No.2, March 2019

$\mathbf{D}_{z}^{*} \mathbf{D}_{z}^{\mathrm{H}}\left\{\sum_{z=1}^{K}\left(\mathbf{H}_{z}^{(U L)}\right)^{*}\left(\mathbf{H}_{Z}^{(U L)}\right)^{\mathrm{H}} \mathbf{P}_{z}^{*}\right\}+\mathbf{D}_{z} \mathbf{D}_{z}^{\mathrm{H}}\left\{\sum_{z=1}^{K} \mathbf{H}_{Z}^{(U L)}\left(\mathbf{H}_{Z}^{(U L)}\right)^{\mathrm{H}} \mathbf{P}_{z}\right\}+2 \mu^{(U L)} k \sum_{z=1}^{K} \mathbf{P}_{z}=2\left(\mathbf{H}_{Z}^{(U L)}\right)^{\mathrm{H}} \mathbf{D}_{z}^{\mathrm{H}}$

where $k=\left[\operatorname{Tr}\left(\sum_{z=1}^{K} \mathbf{P}_{z} \mathbf{P}_{z}^{\mathrm{H}}\right)^{p}\right]^{(1 / p)-1}\left[\sum_{z=1}^{K} \mathbf{P}_{z}^{\top} \mathbf{P}_{z}^{*}\right]^{p-1}$

The expression of Lagrange multiplier is obtained by equating the equations (7) and (8).

$$
\mu^{(U L)}=\frac{\left(\sigma_{n}^{(U L)}\right)^{2}}{2 \alpha}\left[\sum_{z=1}^{K} \operatorname{Tr}\left(\mathbf{D}_{z} \mathbf{D}_{z}^{\mathrm{H}}\right)\right]
$$

An iterative procedure shown in Figure 2 is used to find the optimum solution for $\mathbf{D}_{z}$ and $\mathbf{P}_{z}$

$$
\begin{aligned}
& \mathbf{D}_{z}=\mathbf{D}_{z, R e}+j \mathbf{D}_{z, I m}, \\
& \mathbf{E}_{z, R e}^{(U L)}+j \mathbf{E}_{z, I m}^{(U L)}=\mathbf{D}_{z}\left\{\sum_{z=1}^{K} \mathbf{H}_{Z}^{(U L)} \mathbf{P}_{z} \mathbf{P}_{z}^{\mathrm{H}} \mathbf{H}_{z}^{(U L)^{\mathrm{H}}}\right\}, \\
& \mathbf{F}_{z, R e}^{(U L)}+j \mathbf{F}_{z, I m}^{(U L)}=\left\{\sum_{z=1}^{K}\left(\mathbf{H}_{Z}^{(U L)}\right)^{*} \mathbf{P}_{z}^{*} \mathbf{P}_{z}^{\mathrm{H}}\left(\mathbf{H}_{Z}^{(U L)}\right)^{\mathrm{H}}\right\}, \\
& \mathbf{G}_{z, R e}^{(U L)}+j \mathbf{G}_{z, I m}^{(U L)}=2 \mathbf{P}_{z}^{\mathrm{H}}\left(\mathbf{H}_{Z}^{(U L)}\right)^{\mathrm{H}} .
\end{aligned}
$$

Then $\mathbf{D}_{z, R e}$ and $\mathbf{D}_{z, I m}$ can be expressed as

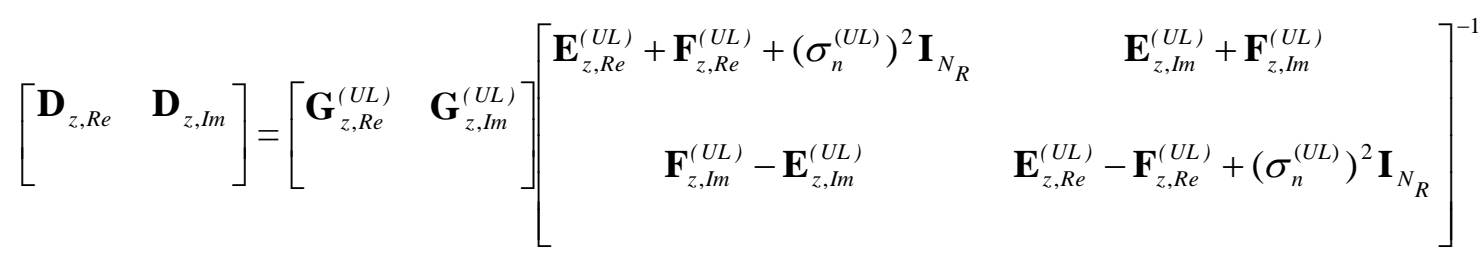

Likewise, define

$$
\begin{aligned}
& \mathbf{P}_{z}=\mathbf{P}_{z, R e}+j \mathbf{P}_{z, I m}, \\
& \mathbf{R}_{z, R e}^{(U L)}+j \mathbf{R}_{z, I m}^{(U L)}=\mathbf{D}_{z} \mathbf{D}_{z}^{\mathrm{H}}\left\{\sum_{z=1}^{K} \mathbf{H}_{Z}^{(U L)}\left(\mathbf{H}_{Z}^{(U L)}\right)^{\mathrm{H}}\right\}, \\
& \mathbf{S}_{z, R e}^{(U L)}+j \mathbf{S}_{z, I m}^{(U L)}=\sum_{m=1}^{K}\left(\mathbf{H}_{Z}^{(U L)}\right)^{\mathrm{H}} \mathbf{D}_{z}^{*} \mathbf{D}_{z}^{\mathrm{H}}\left(\mathbf{H}_{z}^{(U L)}\right)^{*} \\
& \mathbf{T}_{z, R e}^{(U L)}+j \mathbf{T}_{z, I m}^{(U L)}=2\left(\mathbf{H}_{z}^{(U L)}\right)^{\mathrm{H}} \mathbf{D}_{z}^{\mathrm{H}} .
\end{aligned}
$$

Then $\mathbf{P}_{z, R e}$ and $\mathbf{P}_{z, I m}$ can be expressed as

$$
\left[\begin{array}{c}
\mathbf{P}_{z, R e} \\
\mathbf{P}_{z, I m}
\end{array}\right]=\left[\begin{array}{cc}
\left(\mathbf{R}_{z, R e}^{(U L)}+\mathbf{S}_{z, R e}^{(U L)}+2 \mu^{(U L)} k \mathbf{I}_{N, T}\right. & \mathbf{S}_{z, I m}^{(U L)}-\mathbf{R}_{z, I m}^{(U L)} \\
\mathbf{R}_{z, I m}^{(U L)}+\mathbf{S}_{z, I m}^{(U L)} & \mathbf{R}_{z, R e}^{(U L)}-\mathbf{S}_{z, R e}^{(U L)}+2 \mu^{(U L)} k \mathbf{I}_{N, T}
\end{array}\right]^{-1}\left[\begin{array}{c}
\mathbf{T}_{z, R e}^{(U L)} \\
\mathbf{T}_{z, I m}^{(U L)} \\
\end{array}\right]
$$


International Journal of Computer Networks \& Communications (IJCNC) Vol.11, No.2, March 2019

\subsection{Problem formulation for minimum TMSE design with imperfect CSI}

For imperfect case, the $m^{\text {th }}$ user channel is modeled as

$$
\mathbf{H}_{m}^{(U L)}=\mathbf{H}_{m}^{\wedge(U L)}+\mathbf{E}_{m}^{(U L)}
$$

To estimate the channel matrix, training sequences were used.

$$
\mathbf{H}_{m}^{\wedge}(U L)=\mathbf{R}_{R, m}^{1 / 2} \mathbf{H}_{w m}^{\wedge}(U L) \mathbf{R}_{T}^{1 / 2} \text { and } \mathbf{E}_{m}^{(U L)}=\mathbf{R}_{e, R, m}^{1 / 2} \mathbf{E}_{w, m}^{(U L)} \mathbf{R}_{T}^{1 / 2}, \mathrm{~m}=1 \ldots \mathrm{K}
$$

where $\mathbf{R}_{T}^{1 / 2} \& \mathbf{R}_{R, m}^{1 / 2}$ are the correlation matrices and $\mathbf{E}_{m}^{(U L)}$ is the error model. The entries of $\mathbf{H}_{w m}^{\wedge(U L)}$ and $\mathbf{E}_{w, m}^{(U L)}$ are independent and identically distributed.

Substitute equation (13) in (12) to get an expanded version of (12).

$$
\mathbf{H}_{m}^{(U L)}=\mathbf{R}_{R, m}^{1 / 2} \mathbf{H}_{w m}^{\wedge(U L)} \mathbf{R}_{T}^{1 / 2}+\mathbf{R}_{e, R, m}^{1 / 2} \mathbf{E}_{w, m}^{(U L)} \mathbf{R}_{T}^{1 / 2}
$$

where $\mathbf{R}_{e, R, m}=\left[\mathbf{I}_{N_{R, m}}+\sigma_{c e, m}^{2} \mathbf{R}_{R, m}^{-1}\right]^{-1}$ and $\sigma_{c e, m}^{2}=\operatorname{Tr}\left(\mathbf{R}_{T}^{-1}\right) \sigma_{n}^{2} / \mathbf{P}_{t r, m} . \operatorname{In} \mathbf{R}_{e, R, m}, \sigma_{c e, m}^{2}$ is a channel estimation error variance and in $\sigma_{c e, m}^{2}, \sigma_{n}^{2}$ is a noise variance and $\mathbf{P}_{t r, m}$ is a training power of the user $\mathrm{m}$.

The TMSE calculation for the improper modulation based uplink MU-MIMO system for imperfect case defined as follows:

$$
E\left[\left\|\mathbf{e}^{(U L)}\right\|^{2}\right]=E\left[\left\|\mathbf{d}_{l}^{\wedge(U L)}-\mathbf{a}_{l}\right\|^{2}\right]
$$

where $\mathbf{d}_{l}^{\wedge(U L)}=R\left(\mathbf{D}_{l}\left[\sum_{m=1}^{K}\left(\mathbf{H}_{m}^{\wedge(U L)}+\mathbf{E}_{m}^{(U L)}\right) \mathbf{P}_{m} \mathbf{a}_{m}\right]+\mathbf{D}_{l} \mathbf{n}_{l}^{(U L)}\right)$ is the vector after decoder.

Substituting the value of $\mathbf{d}_{l}^{\wedge(U L)}$ in (15), we obtain

$$
E\left[\left\|\mathbf{e}^{(U L)}\right\|^{2}\right]=E\left[\left\|R\left(\mathbf{D}_{l}\left[\sum_{m=1}^{K}\left(\mathbf{H}_{m}^{\wedge(U L)}+\mathbf{E}_{m}^{(U L)}\right) \mathbf{P}_{m} \mathbf{a}_{m}\right]+\mathbf{D}_{l} \mathbf{n}_{l}^{(U L)}\right)-\mathbf{a}_{l}\right\|^{2}\right]
$$

The TMSE calculation is expanded as follows:

$$
\begin{aligned}
& E\left[\left\|\mathbf{e}^{(U L)}\right\|^{2}\right]=\operatorname{Tr}\left\{E \left(0.5\left(\mathbf{D}_{l} \sum_{m=1}^{K} \mathbf{H}_{m}^{\wedge(U L)} \mathbf{P}_{m} \mathbf{a}_{m}+\mathbf{D}_{l}^{*} \sum_{m=1}^{K}\left(\mathbf{H}_{m}^{\wedge(U L)}\right)^{*} \mathbf{P}_{m}^{*} \mathbf{a}_{m}^{*}\right)\right.\right. \\
& \left.+0.5\left(\mathbf{D}_{l} \mathbf{E}_{l}^{(U L)} \sum_{m=1}^{K} \mathbf{P}_{m} \mathbf{a}_{m}+\mathbf{D}_{l}^{*}\left(\mathbf{E}_{l}^{(U L)}\right)^{*} \sum_{m=1}^{K} \mathbf{P}_{m}^{*} \mathbf{a}_{m}^{*}\right)+0.5\left(\mathbf{D}_{l} \mathbf{n}_{l}^{(U L)}+\mathbf{D}_{l}^{*}\left(\mathbf{n}_{l}^{(U L)}\right)^{*}\right)-\sum_{m=1}^{K} \mathbf{a}_{m}\right) \\
& \left(0.5\left(\mathbf{D}_{l}^{\mathrm{H}} \sum_{m=1}^{K}\left(\mathbf{H}_{m}^{\wedge}(U L)\right)^{\mathrm{H}} \mathbf{P}_{m}^{\mathrm{H}} \mathbf{a}_{m}^{\mathrm{H}}+\mathbf{D}_{l}^{\top} \sum_{m=1}^{K}\left(\mathbf{H}_{m}^{\wedge}(U L)\right)^{\top} \mathbf{P}_{m}^{\top} \mathbf{a}_{m}^{\top}\right)+0.5\left(\mathbf{D}_{l}^{\mathrm{H}}\left(\mathbf{E}_{l}^{(U L)}\right)^{\mathrm{H}} \sum_{m=1}^{K} \mathbf{P}_{m}^{\mathrm{H}} \mathbf{a}_{m}^{\mathrm{H}}\right.\right. \\
& \left.\left.+\mathbf{D}_{l}^{\top}\left(\mathbf{E}_{l}^{(U L)}\right)^{\top} \sum_{m=1}^{K} \mathbf{P}_{m}^{\top} \mathbf{a}_{m}^{\top}\right)+0.5\left(\mathbf{D}_{l}^{\mathrm{H}}\left(\mathbf{n}_{l}^{(U L))^{\mathrm{H}}}+\mathbf{D}_{l}^{\top}\left(\mathbf{n}_{l}^{(U L)}\right)^{\top}\right)-\sum_{m=1}^{K} \mathbf{a}_{m}^{\mathrm{H}}\right)\right\}
\end{aligned}
$$

Using the following assumptions made in [15-18] simplifies the equation (17). Then TMSE is calculated as 
International Journal of Computer Networks \& Communications (IJCNC) Vol.11, No.2, March 2019

$$
\begin{aligned}
& E\left[\left\|\mathbf{e}^{(U L)}\right\|^{2}\right]=\operatorname{Tr}\left\{0.25 \mathbf{D}_{l}\left[\sum_{m=1}^{K} \mathbf{H}_{m}^{\wedge}(U L) \mathbf{P}_{m} \mathbf{P}_{m}^{\mathrm{H}}\left(\mathbf{H}_{m}^{\wedge}(U L)\right)^{\mathrm{H}}\right] \mathbf{D}_{l}^{\mathrm{H}}-0.5 \mathbf{D}_{l}^{*} \sum_{m=1}^{K}\left(\mathbf{H}_{m}^{\wedge(U L)}\right)^{*} \mathbf{P}_{m}^{*}\right. \\
& +0.25 \mathbf{D}_{l}\left[\sum_{m=1}^{K} \mathbf{H}_{m}^{\wedge}(U L) \mathbf{P}_{m} \mathbf{P}_{m}^{\top}\left(\mathbf{H}_{m}^{\wedge}(U L)\right)^{\top}\right] \mathbf{D}_{l}^{\top}-0.5 \mathbf{D}_{l}^{\mathrm{H}} \sum_{m=1}^{K}\left(\mathbf{H}_{m}^{\wedge}(U L)\right)^{\mathrm{H}} \mathbf{P}_{l}^{\mathrm{H}} \\
& +0.25 \mathbf{D}_{l} \mathbf{R}_{e, R, l} \mathbf{D}_{l}^{\mathrm{H}}\left[\sum_{m=1}^{K} \operatorname{Tr}\left(\mathbf{R}_{T} \mathbf{P}_{m} \mathbf{P}_{m}^{\mathrm{H}}\right)\right] \sigma_{c e, l}^{2}-0.5 \mathbf{D}_{l} \sum_{m=1}^{K} \mathbf{H}_{m}^{\wedge(U L)} \mathbf{P}_{m}+0.25 \mathbf{D}_{l} \mathbf{D}_{l}^{\mathrm{H}}\left(\sigma_{n}^{(U L)}\right)^{2} \\
& -0.5 \mathbf{D}_{l}^{\top} \sum_{m=1}^{K}\left(\mathbf{H}_{m}^{\wedge}(U L)\right)^{\top} \mathbf{P}_{l}^{\top}+\mathbf{I}_{B_{l}}+0.25 \mathbf{D}_{l}^{*}\left[\sum_{m=1}^{K}\left(\mathbf{H}_{m}^{\wedge}(U L)\right)^{*} \mathbf{P}_{m}^{*} \mathbf{P}_{m}^{\mathrm{H}}\left(\mathbf{H}_{m}^{\wedge(U L)}\right)^{\mathrm{H}}\right] \mathbf{D}_{l}^{\mathrm{H}} \\
& +0.25 \mathbf{D}_{l}^{*}\left[\sum_{m=1}^{K}\left(\mathbf{H}_{m}^{\wedge}(U L)\right)^{*} \mathbf{P}_{m}^{*} \mathbf{P}_{m}^{\top}\left(\mathbf{H}_{m}^{\wedge}(U L)\right)^{\top}\right] \mathbf{D}_{l}^{\top}+0.25 \mathbf{D}_{l}^{*} \mathbf{R}_{e, R, l}^{*} \mathbf{D}_{l}^{\top}\left[\sum_{m=1}^{K} \operatorname{Tr}\left(\mathbf{R}_{T} \mathbf{P}_{m} \mathbf{P}_{m}^{\mathrm{H}}\right)\right]^{*} \sigma_{c e, l}^{2} \\
& +0.25 \mathbf{D}_{l}^{*} \mathbf{D}_{l}^{\top}\left(\sigma_{n}^{(U L)}\right)^{2}
\end{aligned}
$$

To minimize the TMSE subject to PAPC, hence

$$
\min _{\mathbf{P}_{\mathbf{m}}, \mathbf{D}_{\mathbf{m}}} \operatorname{tr}\left(E\left[\left\|\mathbf{e}^{(U L)}\right\|^{2}\right]\right) \text { s.t. } \sum_{m=1}^{K}\left(\operatorname{Tr}\left(\mathbf{P}_{m} \mathbf{P}_{m}^{\mathrm{H}}\right)^{p}\right)^{1 / p} \leq \alpha
$$

Where $\alpha$ is the PAPC.

Using Lagrangian to arrive at the solution for the defined problem

$$
\eta^{(U L)}=E\left[\left\|\mathbf{e}^{(U L)}\right\|^{2}\right]+\mu^{(U L)}\left(\left[\sum_{m=1}^{K}\left(\operatorname{Tr}\left(\mathbf{P}_{m} \mathbf{P}_{m}^{\mathrm{H}}\right)^{p}\right)^{1 / p}\right]-\alpha\right)
$$

where $\mu^{(U L)}$ is the Lagrange multiplier.

By taking the derivatives of $\eta$ with respect to $\mathbf{D}_{l} \& \mathbf{P}_{l}$, it can be shown that the Lagrangian can be derived.

$$
\begin{aligned}
& \frac{\partial \eta^{(U L)}}{\partial \mathbf{D}_{l}}=0 \\
& \mathbf{D}_{z}\left(\sum_{z=1}^{K} \mathbf{H}_{Z}^{\wedge(U L)} \mathbf{P}_{z} \mathbf{P}_{z}^{\mathrm{H}}\left(\mathbf{H}_{z}^{\wedge}(U L)\right)^{\mathrm{H}}+\mathbf{R}_{e, R, z} \sigma_{c e, z}^{2} \mathbf{R}_{T} \sum_{z=1}^{K} \operatorname{Tr}\left(\mathbf{P}_{z} \mathbf{P}_{z}^{H}\right)\right)+\mathbf{D}_{z}^{*} \sum_{z=1}^{K}\left(\mathbf{H}_{Z}^{\wedge}(U L)\right)^{*} \mathbf{P}_{z}^{*} \mathbf{P}_{z}^{\mathrm{H}}\left(\mathbf{H}_{Z}^{\wedge}(U L)\right)^{\mathrm{H}} \\
& +\left(\sigma_{n}^{(U L)}\right)^{2} \mathbf{D}_{z}=2 \mathbf{P}_{z}^{\mathrm{H}}\left(\mathbf{H}_{Z}^{\wedge}(U L)\right)^{\mathrm{H}} \\
& \frac{\partial \eta^{(U L)}}{\partial \mathbf{P}_{l}}=0 \\
& \mathbf{P}_{z}\left(\left(\mathbf{H}_{Z}^{\wedge(U L)}\right)^{\mathrm{H}} \sum_{z=1}^{K} \mathbf{D}_{z} \mathbf{D}_{z}^{\mathrm{H}} \mathbf{H}_{Z}^{\wedge(U L)}+\mathbf{R}_{T} \sigma_{c e, z}^{2} \mathbf{R}_{e, R, z} \sum_{z=1}^{K} \operatorname{Tr}\left(\mathbf{D}_{z} \mathbf{D}_{z}^{H}\right)\right) \mathbf{P}_{z}+\mathbf{P}_{z}^{*}\left(\mathbf{H}_{Z}^{\wedge}(U L)\right)^{\mathrm{H}} \sum_{z=1}^{K} \mathbf{D}_{z}^{*} \mathbf{D}_{z}^{\mathrm{H}}\left(\mathbf{H}_{z}^{\wedge}(U L)\right)^{*} \\
& +2 k \mu^{(U L)} \sum_{z=1}^{K} \mathbf{P}_{z}=2\left(\mathbf{H}_{Z}^{\wedge}(U L)\right)^{\mathrm{H}} \mathbf{D}_{z} \\
& \text { where } k=\left[\operatorname{Tr}\left(\sum_{z=1}^{K} \mathbf{P}_{z} \mathbf{P}_{z}^{\mathrm{H}}\right)^{p}\right]^{(1 / p)-1}\left[\sum_{z=1}^{K} \mathbf{P}_{z}^{\top} \mathbf{P}_{z}^{*}\right]^{p-1}
\end{aligned}
$$

The expression of Lagrange multiplier is obtained by equating the equations (21) and (22). 


$$
\mu^{(U L)}=\frac{\left(\sigma_{n}^{(U L)}\right)^{2}}{2 \alpha}\left[\sum_{z=1}^{K} \operatorname{Tr}\left(\mathbf{D}_{z} \mathbf{D}_{z}^{\mathrm{H}}\right)\right]
$$

An iterative procedure is shown in Figure.6. is used to find the optimum solution for $\mathbf{D}_{z}$ and $\mathbf{P}_{z}$

$$
\begin{aligned}
& \mathbf{D}_{z}=\mathbf{D}_{z, R e}+j \mathbf{D}_{z, I m}, \\
& \mathbf{E}_{z, R e}^{(U L)}+j \mathbf{E}_{z, I m}^{(U L)}=\mathbf{H}_{Z}^{\wedge(U L)} \sum_{z=1}^{K} \mathbf{P}_{z} \mathbf{P}_{z}^{\mathrm{H}}\left(\mathbf{H}_{Z}^{\wedge}(U L)\right)^{\mathrm{H}}+\mathbf{R}_{e, R, z} \sigma_{c e, z}^{2} \mathbf{R}_{T} \sum_{z=1}^{K} \operatorname{Tr}\left(\mathbf{P}_{z} \mathbf{P}_{z}^{\mathrm{H}}\right), \\
& \mathbf{F}_{z, R e}^{(U L)}+j \mathbf{F}_{z, I m}^{(U L)}=\left(\mathbf{H}_{Z}^{\wedge}(U L)\right)^{*} \sum_{z=1}^{K} \mathbf{P}_{z}^{*} \mathbf{P}_{z}^{\mathrm{H}}\left(\mathbf{H}_{Z}^{\wedge}(U L)\right)^{\mathrm{H}}, \\
& \mathbf{G}_{z, R e}^{(U L)}+j \mathbf{G}_{z, I m}^{(U L)}=2 \mathbf{P}_{z}^{\mathrm{H}}\left(\mathbf{H}_{Z}^{\wedge}(U L)\right)^{\mathrm{H}} .
\end{aligned}
$$

Then $\mathbf{D}_{z, R e}$ and $\mathbf{D}_{z, I m}$ can be expressed as

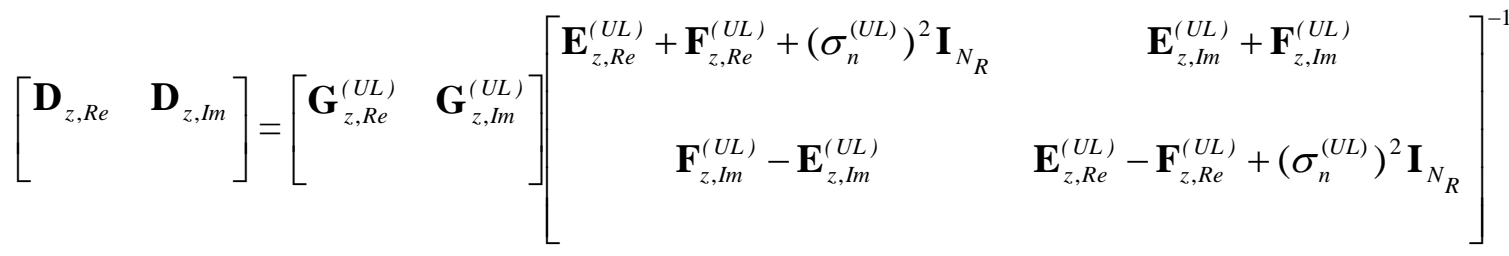

Likewise, define

$$
\begin{aligned}
& \mathbf{P}_{z}=\mathbf{P}_{z, R e}+j \mathbf{P}_{z, I m}, \\
& \mathbf{R}_{z, R e}^{(U L)}+j \mathbf{R}_{z, I m}^{(U L)}=\sum_{z=1}^{K}\left(\mathbf{H}_{z}^{\wedge}(U L)\right)^{\mathrm{H}} \mathbf{D}_{z} \mathbf{D}_{z}^{\mathrm{H}} \mathbf{H}_{z}^{\wedge}{ }_{z}^{(U L)}+\mathbf{R}_{T} \sigma_{c e, z}^{2} \mathbf{R}_{e, R, z} \sum_{z=1}^{K} \operatorname{Tr}\left(\mathbf{D}_{z} \mathbf{D}_{z}^{\mathrm{H}}\right), \\
& \mathbf{S}_{z, R e}^{(U L)}+j \mathbf{S}_{z, I m}^{(U L)}=\sum_{m=1}^{K}\left(\mathbf{H}_{Z}^{\wedge}(U L)\right)^{\mathrm{H}} \mathbf{D}_{m}^{*} \mathbf{D}_{m}^{\mathrm{H}}\left(\mathbf{H}_{Z}^{\wedge}(U L)\right)^{*} \text {, } \\
& \mathbf{T}_{z, R e}^{(U L)}+j \mathbf{T}_{z, I m}^{(U L)}=2\left(\mathbf{H}_{Z}^{\wedge}(U L)\right)^{\mathrm{H}} \mathbf{D}_{z} \text {. }
\end{aligned}
$$

Then $\mathbf{P}_{z, R e}$ and $\mathbf{P}_{z, I m}$ can be expressed as

$$
\left[\begin{array}{c}
\mathbf{P}_{z, R e} \\
\mathbf{P}_{z, I m}
\end{array}\right]=\left[\begin{array}{cc}
\left(\mathbf{R}_{z, R e}^{(U L)}+\mathbf{S}_{z, R e}^{(U L)}+2 \mu^{(U L)} k \mathbf{I}_{N, T}\right. & \mathbf{S}_{z, I m}^{(U L)}-\mathbf{R}_{z, I m}^{(U L)} \\
\mathbf{R}_{z, I m}^{(U L)}+\mathbf{S}_{z, I m}^{(U L)} & \mathbf{R}_{z, R e}^{(U L)}-\mathbf{S}_{z, R e}^{(U L)}+2 \mu^{(U L)} k \mathbf{I}_{N, T}
\end{array}\right]^{-1}\left[\begin{array}{c}
\mathbf{T}_{z, R e}^{(U L)} \\
\mathbf{T}_{z, I m}^{(R L)}
\end{array}\right]
$$

With the help of duality theory, the above design of the Uplink MU-MIMO transceiver system can be implemented for the downlink design. 


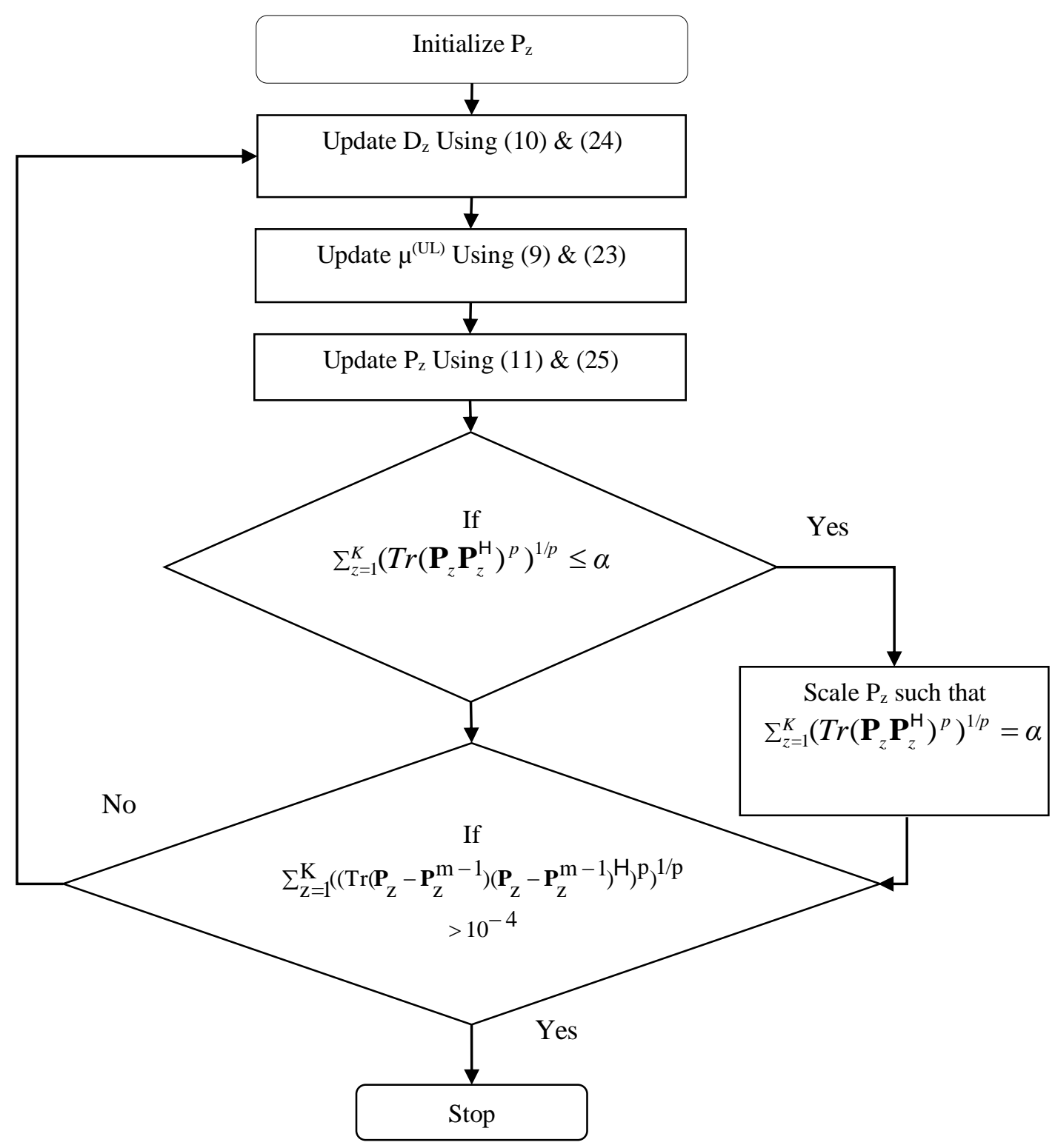

Figure 6. Iterative procedure to get optimum precoder and decoder

\section{RESULTS \&DISCUSSION}

The proposed work aims to improve the security and reliability of MU-MIMO transceiver system. The security of the systems is evaluated by using randomness test, and the reliability of the system is tested in terms of ABER. The proposed cryptographic key generation technique used for security is validated using the National Institute of Standards and Technology (NIST) test suite, and its probability values of the tests are given in Table.2. It is observed that the probability values resulting from all the tests are greater than 0.01. [20] These results show that the generated keys are random in nature. Similarly, the reliability of the proposed MU-MIMO uplink system mathematical model is tested by evaluating the ABER concerning SNR using MATLAB in a simulation environment with parameters described in Table. 3. 
International Journal of Computer Networks \& Communications (IJCNC) Vol.11, No.2, March 2019

Table.2 NIST Parameter analysis

\begin{tabular}{|l|l|l|l|l|l|l|}
\hline Test & P-Key $_{\mathbf{1}}$ & P- Key $_{\mathbf{2}}$ & $\mathbf{P}_{-\mathbf{K e y}_{\mathbf{3}}}$ & $\mathbf{P}_{-\mathbf{K e y}_{\mathbf{4}}}$ & $\mathbf{P}_{-\mathbf{K e y}_{\mathbf{5}}}$ & Result \\
\hline Frequency & 0.534146 & 0.122325 & 0.035174 & 0.350485 & 0.911413 & Pass \\
\hline Block Frequency & 0.534146 & 0.213309 & 0.739918 & 0.739918 & 0.739918 & Pass \\
\hline Cumulative Sums & 0.739918 & 0.122325 & 0.213309 & 0.213309 & 0.534146 & Pass \\
\hline Runs & 0.911413 & 0.122325 & 0.350485 & 0.350485 & 0.350485 & Pass \\
\hline Longest Run & 0.350485 & 0.739918 & 0.017912 & 0.534146 & 0.739918 & Pass \\
\hline Rank & 0.066882 & 0.213309 & 0.534146 & 0.213309 & 0.739918 & Pass \\
\hline FFT & 0.739918 & 0.350485 & 0.534146 & 0.534146 & 0.911413 & Pass \\
\hline $\begin{array}{l}\text { Non-Overlapping } \\
\text { Template }\end{array}$ & 0.991468 & 0.911413 & 0.739918 & 0.911413 & 0.911413 & Pass \\
\hline Approximate Entropy & 0.122325 & 0.122325 & 0.035174 & 0.739918 & 0.739918 & Pass \\
\hline Serial & 0.350485 & 0.534146 & 0.017912 & 0.350485 & 0.739918 & Pass \\
\hline Linear Complexity & 0.350485 & 0.534146 & 0.534146 & 0.534146 & 0.066882 & Pass \\
\hline
\end{tabular}

Table. 3. Simulation parameters and its values

\begin{tabular}{|c|c|c|c|c|c|c|}
\hline Parameter & \multicolumn{6}{|c|}{ Values } \\
\hline Vehicular stations & \multicolumn{6}{|c|}{4} \\
\hline Base station & \multicolumn{6}{|l|}{1} \\
\hline $\begin{array}{l}\text { No of transmitting antennas }\left(\mathrm{N}_{\mathrm{T}}\right) \text { at each } \\
\text { vehicle }\end{array}$ & \multicolumn{6}{|l|}{4} \\
\hline $\begin{array}{l}\text { No of receiving antennas }\left(\mathrm{N}_{\mathrm{R}}\right) \text { at base } \\
\text { station }\end{array}$ & \multicolumn{6}{|l|}{16} \\
\hline No of the data stream (B) from each vehicle & \multicolumn{6}{|l|}{4} \\
\hline \multirow[t]{3}{*}{ p-values } & \multicolumn{2}{|l|}{4.12} & \multicolumn{2}{|l|}{2.36} & \multicolumn{2}{|l|}{1.76} \\
\hline & $\alpha$ & $\beta$ & $\alpha$ & $\beta$ & $\alpha$ & $\beta$ \\
\hline & $1.1 \mathrm{~W}$ & $3.16 \mathrm{~W}$ & $28 \mathrm{~W}$ & $6.31 \mathrm{~W}$ & $5.5 \mathrm{~W}$ & $10 \mathrm{~W}$ \\
\hline
\end{tabular}

The ABER is compared for both proposed and conventional transceiver system, at the output of decryption algorithm by concerning proposed key generation technique. Two improper modulation techniques namely BPSK and 4-ASK modulation are used for evaluation. Standard pvalues such as 4.12, 2.36, and 1.76 are also considered and simulated for both perfect and imperfect CSI condition.

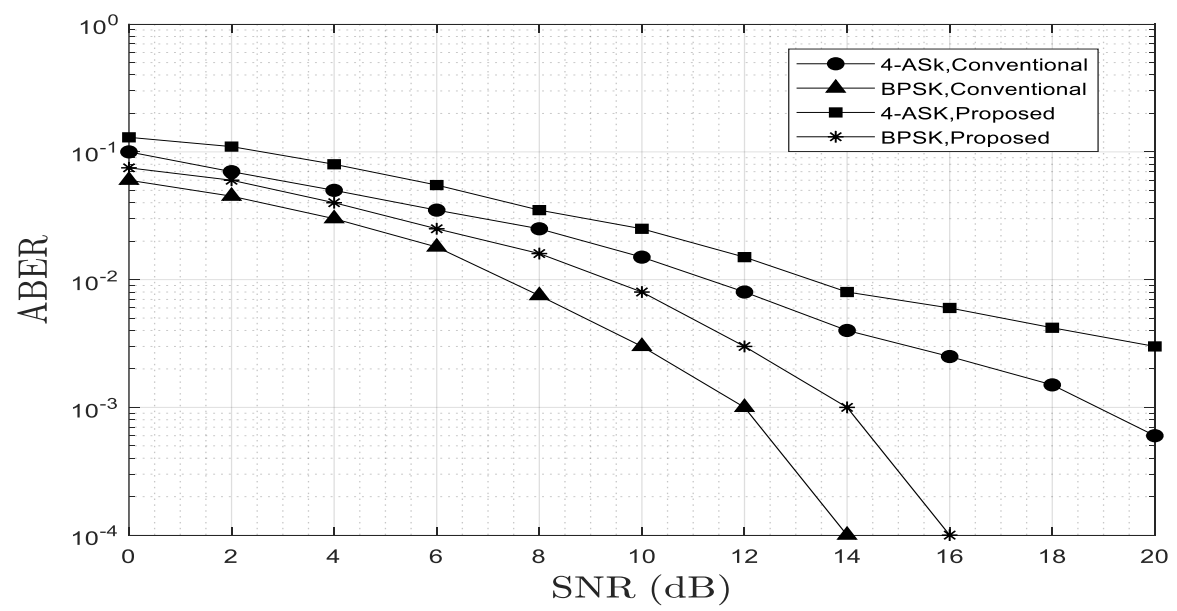

Figure 7. Performance comparison results of MU-MIMO uplink system with respect to perfect CSI for $\mathrm{p}=4.12$ 
Figure 7 shows the performance of the proposed MU-MIMO systems for BPSK and 4-ASK modulation for the p-value of 4.12 with a perfect CSI at the transmitter and receiver. It is observed that SNR is increased from $7.5 \mathrm{~dB}$ to $9.5 \mathrm{~dB}$ for BPSK and from $11.5 \mathrm{~dB}$ to $13.5 \mathrm{~dB}$ in the case of 4-ASK. This indicates that the proposed system demand an additional $2 \mathrm{~dB}$ of SNR at the ABER level of $10^{-2}$. This notifying increase in SNR is worth as the design is more realistic.

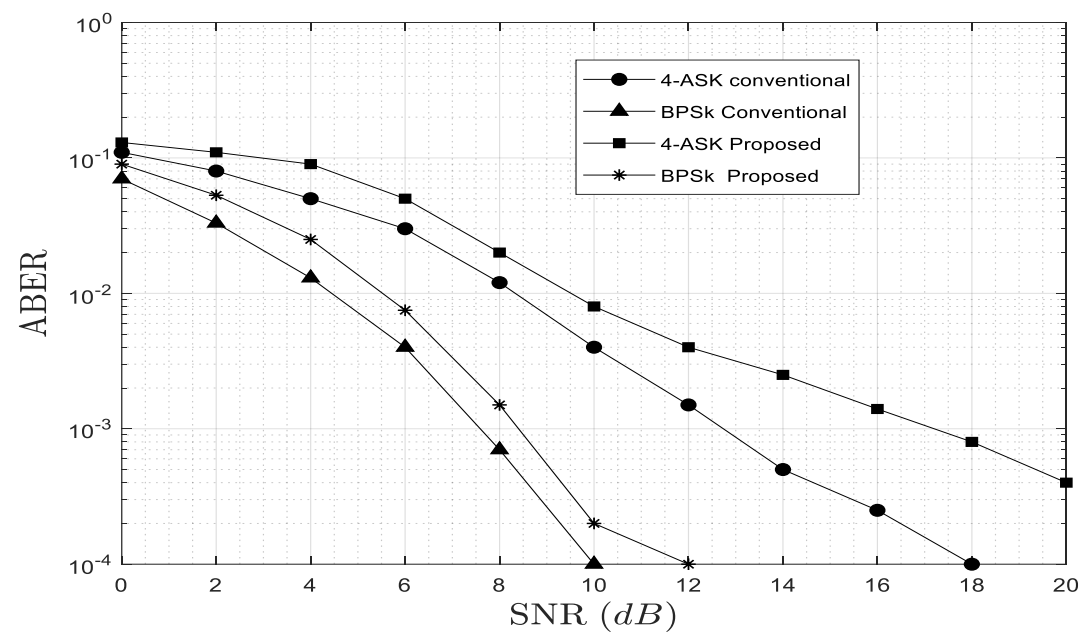

Figure 8. Performance comparison results of MU-MIMO uplink system with respect to perfect CSI for $\mathrm{p}=2.36$

The $p$-value is decreased and simulated for $p=2.36$ as illustrated in Figure 8 . The SNR is increased from $4.5 \mathrm{~dB}$ to $5.5 \mathrm{~dB}$ for BPSK and from $8.5 \mathrm{~dB}$ to $9.5 \mathrm{~dB}$ in the case of 4 -ASK. Here an SNR increase of $1 \mathrm{~dB}$ is observed at the same ABER level of $10^{-2}$ for both the improper modulation schemes. A lesser increase in SNR showcases that the system is nearing optimal as the $\mathrm{p}$-value is reduced from 4.12 to 2.36 .

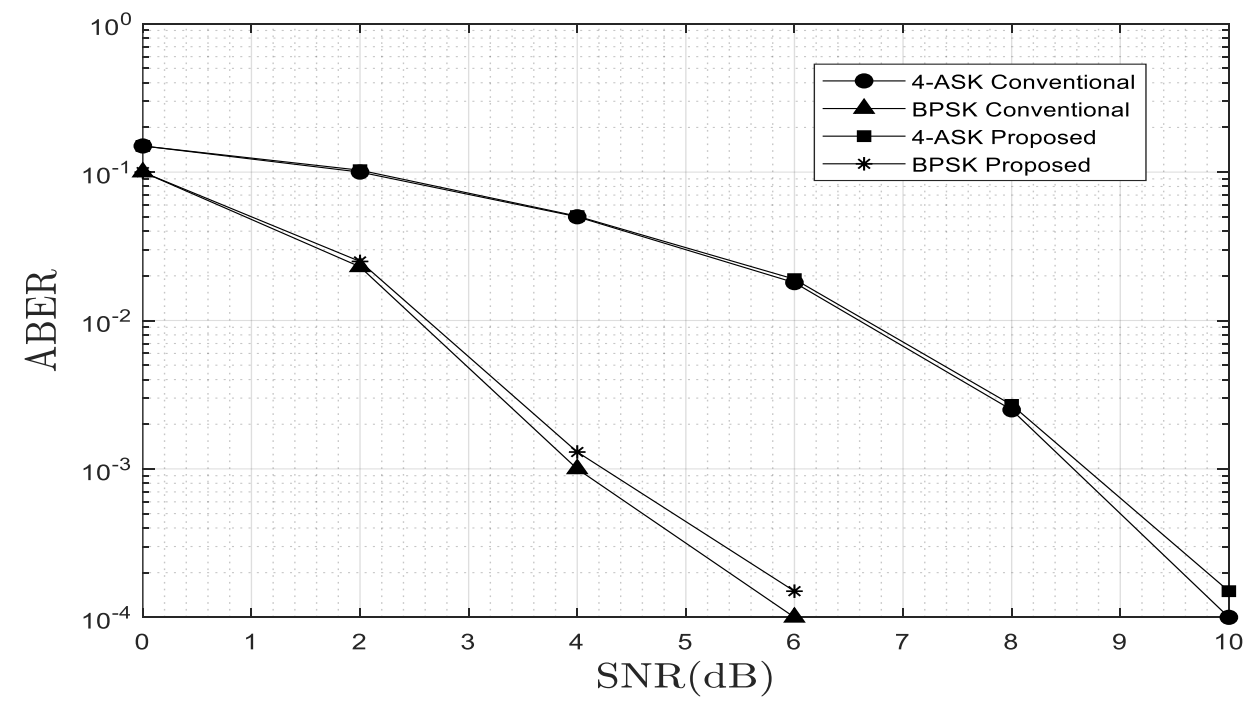

Figure 9. Performance comparison results of MU-MIMO uplink system with respect to perfect CSI for $\mathrm{p}=1.76$

The p-value is further reduced close to unity ( $\mathrm{p}=1.76)$, and its ABER is evaluated as in Figure 9. It is observed that for BPSK modulation, both TPC and PAPC require an SNR value of $2.5 \mathrm{~dB}$ and 4-ASK modulation requires an SNR of $6.5 \mathrm{~dB}$ at $10^{-2} \mathrm{ABER}$. This makes the SNR requirement for PAPC is same as TPC. From these results, the proposed system found to be optimal for practical realization. 
The proposed system is also tested for imperfect CS condition for various $p$-values $(\mathrm{p}=4.12$ and $\mathrm{p}=2.36$ ). The values of transmitting and receive correlation matrices are set to be $\rho_{\mathrm{T}}=0.5, \rho_{\mathrm{R}}=0.5$ respectively and the channel estimation error is set as $\sigma_{\mathrm{ce}}{ }^{2}=0.015$ to simulate the imperfect channel state condition.

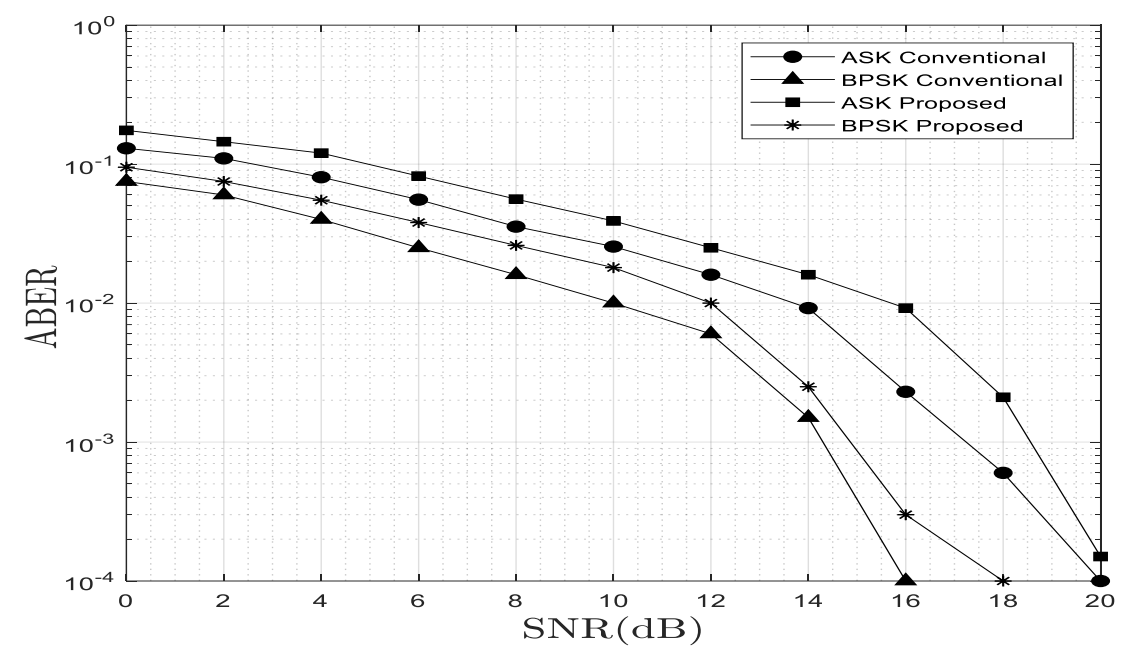

Figure 10a. Performance comparison results of MU-MIMO uplink system with respect to imperfect CSI for $\mathrm{p}=4.12$.

Figure.10a\&10b.shows the comparisons of MU-MIMO uplink system with TPC and PAPC for the imperfect CSI. It illustrates BPSK and 4-ASK requires an increase of $2 \mathrm{~dB}$ (BPSK: 10dB to12 $\mathrm{dB} \&$ 4-ASK: $14 \mathrm{~dB}$ to $16 \mathrm{~dB}$ ) and $1 \mathrm{~dB}$ (BPSK: 7 to $8 \mathrm{~dB} \&$ 4-AKS: $11 \mathrm{~dB}$ to $12 \mathrm{~dB}$ ) for p-value of 4.12 and 2.36 respectively at $10^{-2}$ ABER level. A difference of $2.5 \mathrm{~dB}$ in the SNR is observed between the perfect and imperfect case, which makes the system suitable for realization.

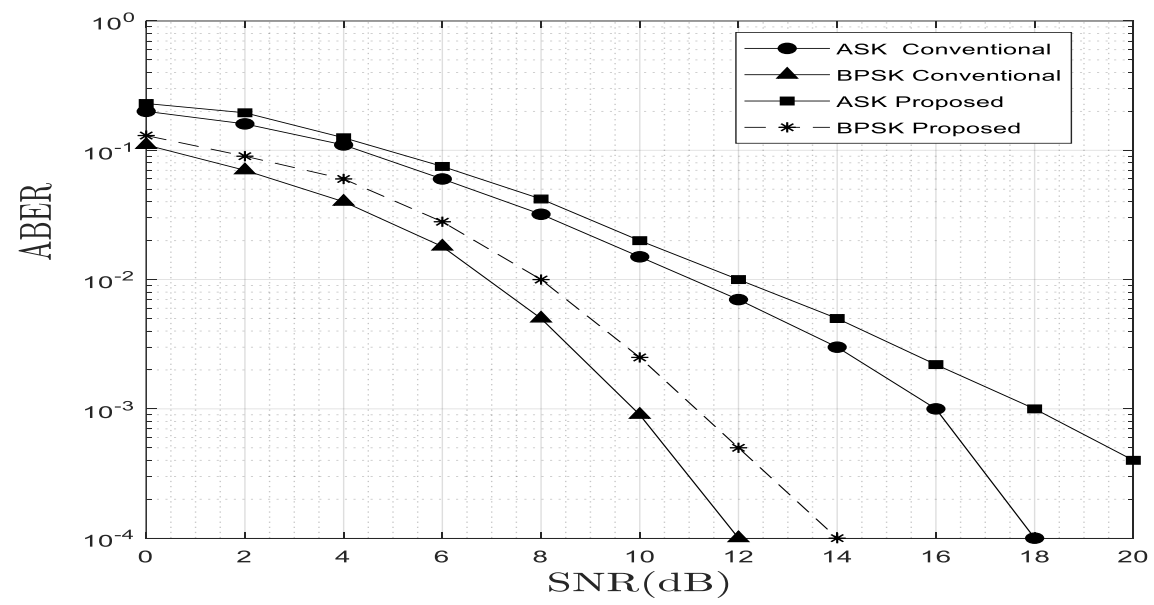

Figure 10b. Performance comparison results of MU-MIMO uplink system with respect to imperfect CSI for $\mathrm{p}=2.36$. 


\section{Conclusions}

In this paper, 1-D improper modulation based uplink MU-MIMO transceiver employing PAPC with TMSE criterion is proposed for the vehicular network to achieve reliability. In addition to that to achieve security, a novel pseudo-random key generation technique was proposed for its stream cipher technique. The proposed key generation technique uses random pixel value based synthetic colour image to generate keys. Five out of 24 generated keys satisfy the randomness test, and its probability value is found to be greater than 0.01 . The proposed key generation techniques make the RF-based vehicular network secure and also the overall transceiver design to be less complex.

Reliability is improved by jointly optimizing the precoder and decoder of the transceiver system using the iterative algorithm. It uses the inverse water-filling technique with respect to PAPC for power allocation, which satisfies the linearity constraint of the power amplifier and makes the system realizable. Simulation studies of the proposed system are carried out for various p-values in the range $1<\mathrm{p}<\infty$ for both perfect and imperfect CS condition. An optimum solution is obtained when the value of $\mathrm{p}$ approaches unity $(\mathrm{p}=1.76)$. Hence the proposed mathematical model for MU-MIMO transceiver system meets the practical requirement of a vehicular network design. It can also be implemented for other RF-MIMO \& visual MIMO communication networks which required low ABER and high security.

\section{ACKNOWLEDGMENTS}

Finally, I would like to thank god for giving me an opportunity, encouragement, and strength to finish this work.

\section{REFERENCES}

[1] Karagiannis, Georgios, Onur Altintas, Eylem Ekici, Geert Heijenk, Boangoat Jarupan, Kenneth Lin, \& Timothy Weil (2011) "Vehicular networking: A survey and tutorial on requirements, architectures, challenges, standards and solutions", IEEE communications surveys \& tutorials, Vol. 13, No. 4, pp584-616.

[2] Saliyah Kahar, Riza Sulaiman, Anton Satria Prabuwono, Nahdatul Akma Ahmad, \& Mohammad Ashri Abu Hassan(2012) " Review of Wireless Technology Usage for Mobile Robot Controller," 2012 International Conference on System Engineering and Modeling, Vol. 34, pp7-12.

[3] Jetmir Haxhibeqiri, Elnaz Alizadeh Jarchlo, Ingrid Moerman, \& Jeroen Hoebeke, (2018) "Flexible Wi-Fi Communication among Mobile Robots in Indoor Industrial Environments," Hindawi Mobile Information Systems, Vol. 2018, pp1-19.

[4] Ningxiao Sun, Yuejin Zhao, Lin Sun, \& Qiongzhi Wu, (2017) "Distributed and Dynamic Resource Management for Wireless Service Delivery to High-Speed Trains," IEEE Access, Vol. 5, pp622-632.

[5] Jankiraman, Mohinder. (2004). Space-time codes and MIMO systems. Artech House

[6] Wong, Kai-Kit. (2004).“Performance analysis of single and multiuser MIMO diversity channels using Nakagami-m distribution," IEEE Transactions on Wireless Communications, Vol.3, No. 4, pp10431047.

[7] Spencer, Quentin H., A. Lee Swindlehurst, \& Martin Haardt. (2004). "Zero-forcing methods for downlink spatial multiplexing in multiuser MIMO channels," IEEE transactions on signal processing, Vol.52, No. 2, pp461-471.

[8] Serbetli, Semih, \& Aylin Yener. (2004),"Transceiver optimization for multiuser MIMO systems." IEEE Transactions on Signal Processing, Vol.52, No. 1, pp241-226. 
[9] Schubert, Martin, Shuying Shi, Eduard A. Jorswieck, \& Holger Boche. (2005). "Downlink sum-MSE transceiver optimization for linear multi-user MIMO systems," Conference Record of the ThirtyNinth Asilomar Conference on Signals, Systems and Computers, pp1424-1428.

[10] Zhang, Jinfan, Yongle Wu, Shidong Zhou, \& Jing Wang. (2005), “ Joint linear transmitter and receiver design for the downlink of multiuser MIMO systems," IEEE communications letters, Vol.9, No. 11, pp991-993.

[11] Shi, Shuying, \& Martin Schubert. (2005), "MMSE transmit optimization for multi-user multi-antenna systems.” IEEE International Conference on Acoustics, Speech, and Signal Processing, pp409-412.

[12] Songnan Xi, \& Michael D. Zoltowski. (2006), “Transmit Beamforming and Detection Design for Uplink Multiuser MIMO Systems, "Fortieth Asilomar Conference on Signals, Systems and Computers, pp. 1593-1600.

[13] Khachan, Ali M., Adam J. Tenenbaum, \& Raviraj S. Adve. (2006). "Linear processing for the downlink in multiuser MIMO systems with multiple data streams," IEEE International Conference on Communications,pp4113-4118.

[14] Xiao, Pei, \& Mathini Sellathurai. (2010). "Improved linear transmit processing for single-user and multi-user MIMO communications systems." IEEE Transactions on Signal Processing, Vol.58, No. 3, pp1768-1779.

[15] Raja, M., Muthuchidambaranathan.P, \& Ha H. Nguyen (2013). "Transceiver Design for MIMO Systems with Improper Modulations,” Wireless Personal Communications, Vol. 68, pp265-280.

[16] Raja, M. \& Muthuchidambaranathan.P, (2012). "Joint Precoding and Decoding in MU-MIMO Downlink Systems with Perfect Channel State Information (CSI)." Procedia Technology, Vol.6, pp708-715.

[17] Raja, M. \& Muthuchidambaranathan.P, (2014). "Multiuser MIMO Transceiver Design for Uplink and Downlink with Imperfect CSI,” Wireless Personal Communications, Vol. 75, pp1215-1234.

[18] Raja, M. \& Muthuchidambaranathan.P, (2013). "SVD-Assisted Joint Precoder and Decoder Design for the Uplink of MU-MIMO Systems With Improper Modulation." Wireless Personal Communications, Vol. 73, No. 3, pp1129-1142.

[19] C. Manikandan, P. Neelamegam, A. Srivishnu \& B. Sabari Ganesh, (2015). “Transceiver Design for SU-MIMO System with Improper Modulations Using per Antenna Power Constraint and Perfect CSI,” International Journal of Mathematical Analysis, Vol. 9, No. 31, pp1519 - 1528.

[20] Andrew Rukhin, Juan Soto, James Nechvatal, Miles Smid, Elaine Barker, Stefan Leigh, Mark Levenson, Mark Vangel, David Banks, Alan Heckert,\&James Dray (2010). "A Statistical Test Suite for Random and Pseudorandom Number Generators for Cryptographic Applications" National Institute of Standards and Technology Special Publication 800-22 revision 1a, pp $1-131$.

\section{AUTHORS}

C.Manikandan received his B.E. Degree in Electronics and Communication Engineering from Anna University and M.Tech. Degree in Digital Communication and Networking from SRM University, Currently, he is working as an Assistant Professor-III in School of Electrical and Electronics Engineering, SASTRA, Deemed University. He has a teaching experience of 9 years, and he has published 18 research articles in refereed International Journals He is currently working towards his Ph.D. Degree in SASTRA, Deemed University. His research area is improving security and reliability for Wireless \& Visual MIMO Communication System. He is a Life Member of ISSE.

P.Neelamegam received his M.Sc. Degree from Madras University, Chennai and Ph.D. Degree from Bharathidasan University, Trichy. Currently, he is working as a Professor in the School of Electrical and Electronics Engineering, SASTRA, Deemed University, Thanjavur. He has teaching experience of 45 years, and he has published $80+$ research articles in refereed International journals His research is related to Signal Processing, Smart Instrumentation, Wireless embedded system, and Networking. He has guided 8 Ph.D. students. He is a Life member of ISSE.
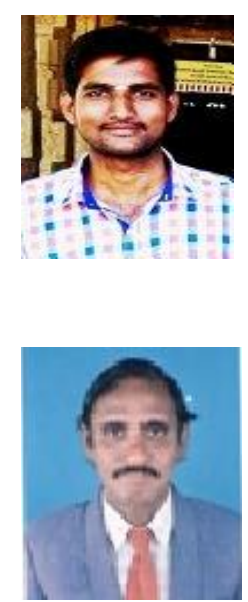
S.Rakesh Kumar received his B.E and M.E in the field of instrumentation engineering. He completed his $\mathrm{Ph} . \mathrm{D}$. in the area of sensor fusion techniques to improve autonomous navigation of wheeled mobile robots. He has nine years of teaching experience and currently working as an assistant professor in the Department of instrumentation engineering, School of electrical and electronics, SASTRA Deemed University, India. He has around 35 research articles published in peer-reviewed international journals. His area of interest includes sensor fusion, robotics and automation, and machine learning techniques.

G.Venkat Babu received his B.E. Degree in Electronics and Communication Engineering from Bharathidasan University, Trichy, M.E. Degree in Microwave communication and RADAR from Dr.B.R.Ambedkar University, Agra and Ph.D. Degree from Bhagwant University, Ajmer. Currently, he is working as an Assistant Professor in School of Electrical and Electronics Engineering, SASTRA, Deemed University, Thanjavur. He has a teaching experience of 16 years, and he has published seven research articles in National and International journals. He Co-authored a book in the field of Electromagnetic Fields. His research area includes Smart Antennas, and RF-MEMS. He is a member of IEEE, Life member of IETE and ISTE.

Sai Siva Satwik Kommi received his Degree in Electronics and Communication Engineering from SASTRA Deemed University, Thanjavur India. He is pursuing masters in Computer and Information Systems Security from Illinois Institute of Technology, Chicago. He has around four research articles published in peer-reviewed international journals and Conferences His research area includes Information Security, Image Steganography and Wireless Communication.
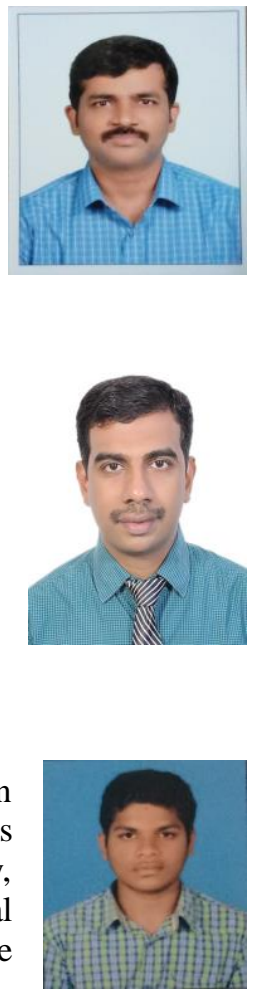